
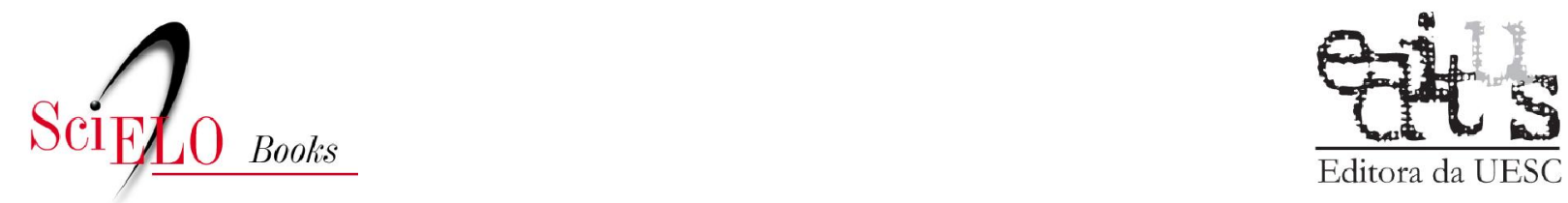

Editora da UESC

\title{
18 - Complexidade e atividade biológica das peçonhas de formigas, em particular de poneromorfas
}

\author{
Renato Fontana \\ Carlos Primino Pirovani \\ Helena Costa \\ Aline Silva \\ Ludimilla Carvalho e Cerqueira Silva \\ Wallace Felipe Blohem Pessoa \\ Juliana Rocha da Silva \\ Jacques H. C. Delabie
}

\section{SciELO Books / SciELO Livros / SciELO Libros}

FONTANA, R., et al. Complexidade e atividade biológica das peçonhas de formigas, em particular de poneromorfas. In: DELABIE, JHC., et al., orgs. As formigas poneromorfas do Brasil [online]. Ilhéus, BA: Editus, 2015, pp. 271-284. ISBN 978-85-7455-441-9. Available from SciELO Books $<\underline{\text { http://books.scielo.org }>\text {. }}$

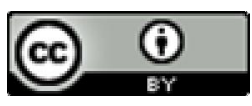

All the contents of this work, except where otherwise noted, is licensed under a Creative Commons Attribution 4.0 International license.

Todo o conteúdo deste trabalho, exceto quando houver ressalva, é publicado sob a licença Creative Commons Atribição 4.0.

Todo el contenido de esta obra, excepto donde se indique lo contrario, está bajo licencia de la licencia Creative Commons Reconocimento 4.0. 


\title{
Complexidade e atividade biológica das peçonhas de formigas, em particular de poneromorfas
}

\author{
Renato Fontana, Carlos Primino Pirovani, Helena Costa, Aline Silva, \\ Ludimilla Carvalho e Cerqueira Silva, Wallace Felipe Blohem Pessoa, \\ Juliana Rocha da Silva, Jacques H. C. Delabie
}

\section{Resumo}

Comparados aos de outros animais peçonhentos, os venenos de formigas são ainda pouco explorados. No Brasil, os acidentes provocados por ferroadas desses insetos, em geral, não são documentados. A partir de pesquisas por artigos na plataforma PubMed, observamos que ainda pouco se sabe sobre as peçonhas de formigas, uma vez que foram encontrados somente cerca de 400 artigos publicados sobre este tema, enquanto há muito mais informação disponível sobre outros tipos de peçonhas, como as de serpentes. As peçonhas de formigas podem ser consideradas como uma fonte ímpar de compostos novos, caracterizados principalmente por proteínas, peptídeos, alcaloides e aminas. Identificar os componentes dessas peçonhas pode ajudar a entender seu mecanismo de ação e a importância de seus constituintes para a sobrevivência das formigas, os mecanismos de sua toxicidade no ser humano e outros organismos, além de auxiliar no desenvolvimento de compostos de interesse estratégico para as indústrias farmacêutica e química em geral, visando o bem-estar da população. Para alcançar isto, os pesquisadores estão utilizando tecnologias de diferentes áreas do conhecimento científico, principalmente com a finalidade de descobrir e desenvolver novos fármacos. As técnicas utilizadas em análise proteômica estão auxiliando a desvendar a composição proteica e a atividade biológica dos constituintes dos venenos. Do ponto de vista da toxinologia molecular e da venômica, o interesse científico para peçonhas animais tem crescido acentuadamente devido à variedade de compostos bioativos já descobertos, aos riscos para a segurança, e aos usos terapêuticos potenciais de compostos isolados. Os resultados apontam para uma grande diversidade estrutural dos peptídeos, com uma estimativa de que mais de uma centena de peptídeos diferentes já seja conhecida na composição das peçonhas. Encontramos, também, grande quantidade de enzimas, tais como lípases, hialuronidases, fosfatases, fosfolipases e esterases. Peçonhas de formigas que possuem grande quantidade de alcaloides na sua composição foram bem estudadas até o momento, principalmente em espécies dos gêneros Monomorium e Solenopsis. No entanto, peçonhas de formigas das mais diversas subfamílias se mostraram ricas em compostos proteicos e peptídicos. Nas poneromorfas isso também é verdade e grande quantidade de peptídeos foi descrita especialmente nas peçonhas de Ectatomminae e de Ponerinae.

FONTANA, Renato; PIROVANI, Carlos Primino; COSTA, Helena; SILVA, Aline; SILVA, Ludimilla Carvalho e Cerqueira; PESSOA, Wallace Felipe Blohem; SILVA, Juliana Rocha da; DELABIE, Jacques H. C. Complexidade e atividade biológica das peçonhas de formigas, em particular de poneromorfas. In: DELABIE, Jacques H. C. et al. As formigas poneromorfas do Brasil. Ilhéus: Editus, 2015. p. 271-284. 
Complexity and biological activity of venoms of ants, particularly poneromorph ants - Compared with other venomous animals, ant poisons are largely unexplored. In Brazil, incidents caused by stings of these insects are generally not documented. From papers found through PubMed, we note that little is known about the venom of ants, with only about 400 articles published on this topic being found. By contrast, there is much more information available on other types of venom, such as that of snakes. Ant venoms can be considered as a source of novel compounds, mainly types of proteins, peptides, amines and alkaloids. Identifying the components of these venoms can help us to understand their mechanism of action, the importance of their constituents to ant survival, the mechanisms of their toxicity to humans and other organisms, and can assist in the development of compounds of strategic interest for the pharmaceutical industry and the field of chemistry in general. To achieve this, researchers are using technologies from different scientific fields, primarily for the purpose of discovering and developing new drugs. The techniques used in proteomics analysis are helping to unravel the protein composition and biological activity of the poison constituents. From the point of view of molecular toxinology and venomics, scientific interest for animal venoms has grown sharply due to the variety of bioactive compounds already discovered, the risks to safety, and the potential therapeutic uses of isolated compounds. The results point to a major structural diversity of peptides, with more than a hundred different peptides already known in the composition of venoms. We also found large amounts of enzymes such as lipases, hyaluronidases, phosphatases, phospholipases and esterases. Venoms of ants that contain alkaloids have been well studied to date, especially in species of the genera Monomorium and Solenopsis. However, ant venoms from different subfamilies have been proven rich in protein and peptide compounds. In poneromorphs this is also true and many peptides have been described, especially in the venoms of Ectatomminae and Ponerinae.

\section{Introdução}

No Brasil em particular, e nas Américas em geral, acidentes sérios provocados por ferroadas de formigas são raramente documentados. Porém, já foram descritas as graves consequências que uma única ferroada pode ocasionar, tais como dor aguda, sudorese, náuseas, alergias, choque anafilático, taquicardia, entre outras manifestações (HADDAD JR. et al., 2005; BLUM, 1992). A "falsa tocandira" (Dinoponera gigantea Perty, 1833), por exemplo, é capaz de aplicar uma ferroada extremamente dolorosa que pode provocar manifestações sistêmicas como febre, tremores, sudorese, náuseas, vômito, linfadenopatia e arritmia cardíaca (HADDAD JR. et al., 2005). Em alguns casos, uma sequência de ferroadas pode levar a vítima à morte (STEEN et al., 2005).

Muitos pesquisadores, juntamente com a indústria, têm buscado integrar diferentes áreas como farmacologia, bioquímica, química e biofísica, além da biologia molecular, no intuito de descobrir moléculas naturais de origem animal que sirvam de protótipo para o desenvolvimento de novos fármacos.
Por isso, a utilização de técnicas moleculares, associada a ensaios biológicos, é de relevância indiscutível na busca de produtos naturais ainda pouco ou não explorados que apresentam compostos bioativos (PUPO; GALLO, 2007). Segundo Touchard et al. (2014), as peçonhas de formigas estão entre as fontes mais abundantes de compostos bioativos do planeta, ainda que praticamente inexploradas.

\section{Métodos de Estudos de Peçonhas}

A atividade das peçonhas de formigas é conhecida desde o final do século XVII (SPERLING, 1689, In: WHEELER, 1910), mas, comparada com a de outros animais, ainda pouco se sabe sobre sua composição, propriedades farmacológicas, imunológicas e alergênicas. Pinto et al. (2012) descrevem as peçonhas de formigas como ferramentas químicas, estratégicas na defesa das colônias, captura das presas, controle do território contra outros animais e na higienização contra doenças no interior da colônia.

Um dos motivos da carência de estudos realizados com peçonhas de formigas é a pequena 
quantidade de material biológico que pode ser extraído dos reservatórios glandulares (SANTOS et al., 2011), o que muito dificulta a identificação de componentes e análise de atividades farmacológicas. Contudo, na plataforma PubMed (Biblioteca Nacional de Saúde e Medicina dos Estados Unidos), vinculada ao NCBI (Centro Nacional de Informação em Biotecnologia dos Estados unidos), são encontrados atualmente 396 artigos (http://www.ncbi. nlm.nih.gov/pubmed/ consultado em 04/02/2015), quando se combinam as palavras-chaves "venom" e "ant". Segundo esse site, a primeira publicação abordando explicitamente o tema é de 1946 e 83\% das publicações ocorreram nos últimos 30 anos com uma forte tendência a aumentar (Figura 18.1). Comparativamente, quando a palavra-chave ant é substituída por snake (serpente), são listadas 12.300 publicações, ou seja, até os tempos atuais, o investimento no estudo de venenos de serpentes foi cerca 31 vezes maior do que em relação aos venenos de formigas. Isto pode estar relacionado à importância toxicológica, à relativa facilidade de coleta do veneno das serpentes e à quantidade disponível por indivíduo, associando apelo e facilidade de estudo.

\section{Proteômica de Peçonhas}

O termo Proteômica foi empregado pela primeira vez em 1995 e definido como a caracterização em larga escala do conjunto de proteínas (o proteoma) expressas em uma célula ou tecido, a partir do genoma (WILKINS et al., 1996). O proteoma de um organismo reflete o conjunto de proteínas expressas por ele em determinada situação e, ao contrário do genoma, não é estático e pode se modificar, dependendo das condições e estímulos às quais este organismo está exposto (SILVA e SILVA et al., 2007). A proteômica estuda a estrutura, a função e o controle dos sistemas biológicos pela análise das propriedades, funções, sequência (identidade), abundância e atividade das proteínas expressas por uma célula ou organismo, assim como as modificações, interações e translocações sofridas pelas proteínas (WILLIAMS; HOCHSTRASSER, 1997).

Desta maneira, estudos proteômicos levam a três vertentes básicas, com implicações diretas nos campos da biologia molecular e da biotecnologia: (i) permitem a descoberta de vias metabólicas nas diversas etapas celulares, gerando um conhecimento sem precedente em biologia celular e bioquímica; (ii) viabilizam a identificação de novas moléculas bioativas, levando ao desenvolvimento de novos fármacos; e (iii) permitem também a identificação e caracterização de marcadores biológicos, isto é, moléculas endógenas ou exógenas específicas de determinado estado patológico (ROCHA et al., 2007).

Figura 18.1 Evolução do número de publicações sobre veneno de formigas informadas no PubMed, nos últimos 30 anos. Na busca realizada em http://www.ncbi.nlm.nih.gov/pubmed/ foram utilizadas as palavras-chave "ant" e "venom".

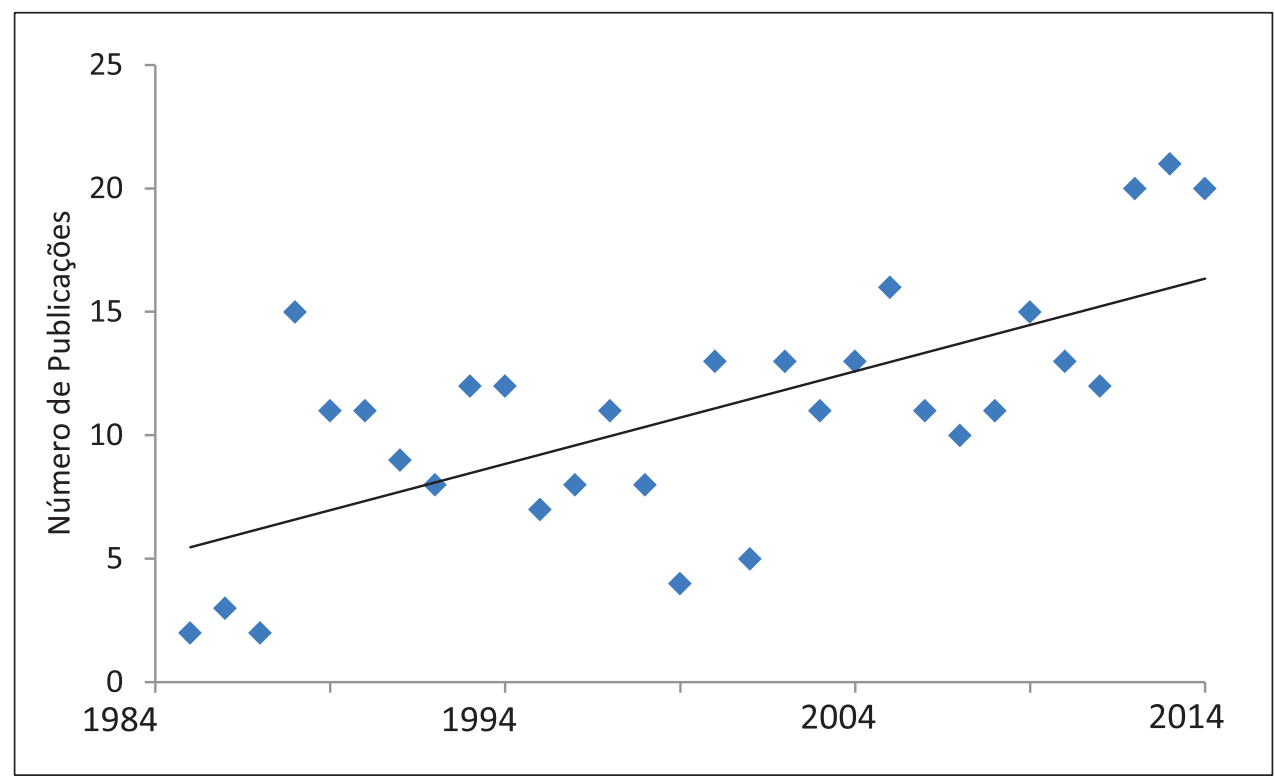


A proteômica tem um papel essencial na descoberta de novos peptídeos e permite aos pesquisadores visualizar, comparar e identificar uma grande quantidade de proteínas. Além disso, possibilita a obtenção de informações quantitativas de proteínas expressas e modificações pós-transducionais que não podem ser deduzidas através do estudo do genoma (DIZ et al., 2012). Vale ressaltar, no entanto, que os bancos de dados genômicos e transcriptômicos facilitam as análises proteômicas, especialmente na identificação de proteínas. O processamento e análise de dados proteômicos é complexo e consiste em uma sucessão de etapas metodológicas (DOMON; AEBERSON, 2006). Uma análise transparente e consistente de informações nas sequências LC/MS (Cromatografia Líquida/ Espectrometria de Massas) ou LC/MS/MS (Cromatografia Líquida/ Espectrometria de Massas/ Espectrometria de Massas) requer múltiplos estágios. Esses incluem desde o processamento de dados brutos para extrair informações e sinais relevantes, a busca em banco de dados para designar os espectros de sequências proteicas, e o armazenamento dos resultados em bancos de dados (DOMON; AEBERSON, 2006).

No banco de dados de proteínas do NCBI existem atualmente 283 acessos para sequências de proteínas de peçonhas de formiga (palavraschave: ant e venon) (http://www.ncbi.nlm.nih. gov/pubmed/ acesso em 04/02/2015). Mas, esse banco de dados pode apresentar redundâncias. Por outro lado, no banco de dados do UNIPROT (http://www.uniprot.org/ acesso em 01/01/2015), onde são depositadas as sequências de proteínas caracterizadas, livres de redundâncias, são encontradas somente 88 sequências. Dessas, 41 foram revisadas e depositadas no banco de dados sobre sequências proteicas do Swiss-Prot (http://web. expasy.org/docs/swiss-prot_guideline.html/ acesso em 20/03/2015) e o restante não foi revisado. A quantidade de dados depositados nestes bancos tem crescido nos últimos anos e isto facilita novas análises proteômicas, que visam à identificação de componentes das peçonhas de formigas. Por sua vez, a Figura 18.1 evidencia um crescimento sensível no número de publicações no NCBI, com mais de 60 publicações $(\sim 16 \%)$ somente nos últimos três anos, contra uma média de 12 publicações por ano nos anos anteriores. Esse recente crescimento do número de publicações sobre os venenos de formiga está certamente relacionado ao emprego das análises proteômicas. Por outro lado, estes dados revelam também um baixo número de pesquisadores dedicados a este tema.

Pesquisas visando identificar componentes das peçonhas de himenópteros podem ajudar a entender melhor o mecanismo de sua ação no organismo humano e podem auxiliar no desenvolvimento de alérgenos recombinantes para usos diagnóstico e terapêutico, a fim de beneficiar pessoas que apresentam reações alérgicas graves a esses compostos (SANTOS; PALMA, 2009; SANTOS et al., 2011). Através de estudos proteômicos, por exemplo, podem-se identificar proteínas alergênicas, proteínas envolvidas no dano tecidual e capazes de promover inflamação de tecidos, algumas neurotoxinas e proteínas associadas à comunicação química que funcionam como feromônios (PINTO et al., 2012). Dessa forma, análises proteômicas ajudam a entender os processos fisiológicos envolvidos no envenenamento, assim como aqueles relacionados mais particularmente à biologia e ecologia do animal nas suas relações com seu habitat e a comunidade de organismos na qual ele se insere.

Assim, do ponto de vista da toxinologia molecular e da venômica, o interesse científico e a preocupação pública sobre peçonhas animais têm crescido acentuadamente devido à grande diversidade de compostos bioativos, aos riscos para a segurança, e aos usos terapêuticos potenciais de compostos isolados (BOUZID et al., 2013).

\section{Venômica: a Proteômica aplicada ao estudo das peçonhas}

Fontes ricas de moléculas bioativas, as peçonhas de diversos animais têm sido exploradas através de técnicas proteômicas. Esta abordagem, desde 2009, é denominada Venômica (ESCOUBAS; KING, 2009).

Até a década de 1990, os estudos baseavam-se em métodos clássicos de caracterização bioquímica, tais como a cromatografia associada à eletroforese unidirecional (SDS-PAGE). Com o advento da Proteômica e o posterior desenvolvimento de técnicas mais sofisticadas, um novo panorama no estudo das peçonhas foi estabelecido. Protocolos como a eletroforese bidimensional (2D-SDS-PAGE) e a espectrometria de massas permitiram um aprofundamento sobre a bioquímica das peçonhas, especialmente no que diz respeito às proteínas (FOX; SERRANO, 2008).

Componentes proteicos da peçonha de diversos animais já foram total ou parcialmente caracterizados. Entre estes organismos, destacam-se 
espécies de aranhas (SANTOS et al., 2009; KUHNNENTWIG et al., 2011), serpentes (GIEORGIEVA et al., 2011), abelhas (GRAAF et al., 2009), vespas (SANTOS et al., 2011), formigas (HOFFMAN et al., 2005), escorpiões (SCHWARTZ et al., 2008), centopeias (RATES et al., 2007) e sapos (RATES et al., 2011).

\section{Complexidade e Estrutura das Peçonhas}

Insetos são invertebrados dotados de uma grande variedade de mecanismos de defesa, o que é um dos motivos pelos quais conseguiram ocupar os mais diversos ambientes terrestres (HÖLLDOBLER; WILSON, 1990; PALMA, 2006). As formigas são himenópteros aculeados, por apresentarem basalmente um aparelho inoculador (ferrão), além das glândulas secretoras correspondentes, onde a peçonha é produzida, armazenada em um reservatório conectado ao aparato inoculador. Algumas subfamílias de Formicidae perderam o ferrão através do processo evolutivo, através de modificações na morfologia da região terminal do gáster (como, por exemplo, o acidóporo na subfamília Formicinae, utilizado para projetar um veneno composto basicamente de acido fórmico e hidrocarbonetos).

\section{i) Hymenoptera não Formicidae}

Há mais de 50 anos, verificou-se que a peçonha bruta de abelhas apresentava atividade antimicrobiana, e a melitina foi isolada como uma das frações responsáveis por essa atividade (FENNEL et al., 1967). A peçonha bruta e uma fração isolada por cromatografia líquida apresentaram atividade antimicrobiana contra bactérias gram-positivas e gram-negativas, inclusive cepas resistentes de Staphylococcus aureus (FENNEL; SHIPMAN; COLE, 1967).

As peçonhas de himenópteros são caracterizadas como uma mistura complexa de proteínas, peptídeos, alcaloides e aminas (HIDER, 1988; JONES et al., 1999; HOFFMAN, 2006; PALMA, 2006; PINTO et al., 2012; TOUCHARD et al., 2014a,b). Os peptídeos são, em sua maioria, catiônicos, pequenos e lineares, apresentando um alto conteúdo de $\alpha$-hélices, que são responsáveis pela lise de células, hemólise, liberação de histamina por mastócitos e atividades antimicrobianas (PALMA, 2006; AILI et al., 2014). Em geral, os peptídeos estão envolvidos em processos que incluem a permeabilização da membrana e sua consequente destruição, ocasionando a morte celular (DESBOIS et al., 2010). Esses processos podem também intensificar a dispersão de outras toxinas da peçonha nos tecidos (BOUZID et al., 2013). Dessa forma, himenópteros oferecem uma infinidade de moléculas biologicamente ativas que incorporam as bases fisiopatológicas das causas das alergias, do envenenamento pelas peçonhas de formigas e pelo uso tradicional e atual de formigas para medicamentos e na procura de novas drogas pela indústria (AILI et al., 2014; TORRES et al., 2014).

\section{ii) Formicidae não poneromorfas}

Touchard et al. (2014a) analisam a composição e a diversidade estrutural de peptídeos nas peçonhas de numerosas formigas com ferrão, poneromorfas ou não, em diversas subfamílias (Paraponerinae, Ponerinae, Myrmeciinae, Pseudomyrmecinae, Ectatomminae, Myrmicinae). Seus resultados apontam a grande diversidade estrutural dos peptídeos presentes na peçonha de formigas e demonstraram, por diversas metodologias que incluem a espectrometria de massa, que existem mais de uma centena de peptídeos diferentes na composição dessas peçonhas. Concluem afirmando que a complexidade de moléculas bioativas presentes na peçonha das formigas é semelhante ou maior que as encontradas em aranhas, escorpiões e serpentes (TOUCHARD et al., 2014a,b). De fato, até a presente data, as peçonhas de apenas onze espécies de formigas foram caracterizadas bioquimicamente e 72 sequências de peptídeos foram descritas, como, por exemplo, as poneratoxinas (PIEK et al., 1991, SZOLAJSKA et al., 2004), as ponericinas (ORIVEL et al., 2001), as bicarinalinas (RIFFLET et al., 2012) e as dinoponeratoxinas (COLOGNA et al., 2013, JOHNSON et al., 2010) Além disso, peçonhas de formigas contêm ainda enzimas variadas, como lipases, hialuronidases, fosfolipases ou estereases (PLUZHNIKOV et al., 2006).

Entre as Dorylinae, a única informação que se tem sobre peçonhas é para a espécie neotropical Eciton burchellii cujo veneno possui forte atividade esterásica, mas apresenta uma moderada imunogenicidade e citotoxicidade (BLUM, 1992).

Única entre as Myrmeciinae australianas, a composição proteica da peçonha de Myrmecia pilosula (Jack jumper ant) foi descrita, utilizando as técnicas de SDS-PAGE, cromatografia líquida de alta eficiência e espectrometria de massas. Estas 
técnicas revelaram que o veneno possui um perfil formado por proteínas variando em tamanho de 26 a $90 \mathrm{kDa}$, dentre elas a pilosulina 1 (ou Myr p 3), uma proteína com caráter alergênico ligante de IgE (PAN; HINK, 2000; WIESE et al., 2006). As pilolusinas compreendem uma família de peptídeos que apresentam atividade hemolítica e citotoxicidade a linfócitos B transformados pelo vírus Epstein-Barr e a células mononucleares normais de sangue periférico (WU et al., 1998).

Na subfamília Myrmicinae há, sobretudo, informações sobre os venenos em três gêneros: Pogonomyrmex, Solenopsis e Tetramorium.

Formigas do gênero Pogonomyrmex apresentam elevado teor de enzimas no veneno, possuindo quantidades significativas de fosfolipases A e B, bem como hialuronidases, lipases, fosfatases e esterases, todas com potencial hemolítico (SCHMIDT; BLUM, 1978a e b).

Entre as "formigas de fogo", Solenopsis invicta é, de longe, a formiga cuja peçonha é a mais bem estudada. Sabe-se, que $95 \%$ do veneno são constituídos por alcaloides, principalmente a piperidina responsável pela pustulação no local da ferroada (DESLIPPE; GUO, 2000; CHEN; FADAMIRO, 2009). Hoffman e colaboradores (1988) identificaram quatro proteínas alergênicas (Sol i 1, Sol i 2, Sol i 3 e Sol i 4) na peçonha de $S$. invicta, sendo que a Sol i 2 e Sol i 4 não são similares às proteínas caracterizadas e encontradas em outros venenos (HOFFMAN; DOVE; JACOBSON, 1988). A Sol i 3 pertence à família do Antígeno 5 e a Sol i 1 é uma fosfolipase (HOFFMAN, 1993; HOFFMAN et al., 2005). Outras espécies do gênero Solenopsis, tais como S. richteri, S. germinata, S. aurea e S. xyloni, possuem peçonhas com composição semelhante à de $S$. invicta, para as quais também foram identificados quatro alérgenos proteicos principais (HOFFMAN et al., 1990).

Em 2005, Valles; Pereira identificaram e caracterizaram a presença da proteína transferrina na peçonha de $S$. invicta. Estudos moleculares da transferrina em várias espécies de insetos indicaram que esta é uma proteína multifuncional, de ligação iônica envolvida no metabolismo do ferro (YUN et al., 2009). Esta glicoproteína, ao controlar o nível de íons livres em fluidos biológicos, desempenha um papel biocida em bactérias, pois mantém o Fe3+ indisponível para ser utilizado no metabolismo microbiano (YUN et al., 2009). A transferrina obtida por processo recombinante possui atividade antimicrobiana contra bactérias gram-positivas e gram-negativas (YUN et al., 2009). Dessa forma, acredita-se que esta proteína pode desempenhar papel importante na defesa contra patógenos invasores e, juntamente com outros peptídeos com ação antimicrobiana, atue na manutenção de assepsia da colônia (PINTO et al., 2012).

A peçonha bruta de Tetramorium bicarinatum possui atividade antibacteriana contra cepas selvagens de Staphylococcus aureus e S. xylosus (BOUZID et al., 2013). Já no veneno de Tetramorium caespitum, foram identificados até o momento predominantemente componentes proteicos desprovidos de atividades fosfolipásica ou hialuronidásica (VON SICARD et al., 1989).

Diversas Myrmicinae possuem peçonha cujos componentes principais são alcaloides do tipo piperidina, pirrolidina e pirrolizínicos, passíveis de serem utilizados para a concepção de novos produtos farmacêuticos (PLUZHNIKOV et al., 2006).

No veneno da Pseudomyrmecinae Pseudomyrmex triplarinus foram encontradas proteínas que foram caracterizadas como fosfolipase, mimerxina e hemolisina (Pan, 2000 Apud: HOFFMAN, 2010). TOUCHARD et al. (2014c) descreveram a ação paralisante e letal da peçonha da Pseudomyrmex penetrator, $P$. termitarius e $P$. gracilis em operários do térmita Nasutitermes costalis. As análises das frações obtidas por HPLC e MALDI-TOF MS revelaram que o veneno de $P$. termitarius é composto de 87 peptídeos lineares, enquanto que as peçonhas de P. gracilis e P. penetrator são compostas por 23 e 26 peptídeos, respectivamente. Os autores defendem que estes peptídeos estão diretamente ligados à ação paralisante e letal destas peçonhas às presas.

\section{iii) Poneromorfas}

Nas Ectatomminae são conhecidas algumas neurotoxinas, como a ectatomina, isolada da peçonha de Ectatomma tuberculatum. Esta é uma proteína básica, de peso molecular igual a 7.928 $\mathrm{Da}$ e ponto isoelétrico igual a 9.95. Em concentrações baixas $(0,05$ a $1,0 \mu \mathrm{M})$, a ectatomina provoca a formação de canais em membranas celulares e artificiais e é responsável pela maior toxicidade da peçonha em mamíferos e insetos (PLUZHNIKOV et al., 1999).

Na subfamília Paraponerinae, a única informação disponível é o isolamento de um neuropeptídeo na peçonha de Paraponera clavata (SZOLAJSKA et al., 2004). 
Entre as Ponerinae, Orivel et al. (2001) isolaram 15 novos peptídeos que apresentaram atividade antimicrobiana, inseticida e hemolítica na peçonha da espécie neotropical Neoponera goeldii. Em Dinoponera australis (Ponerinae), foram identificados seis peptídeos para os quais a atividade biológica ainda não foi definida (JOHNSON et al., 2010, COLOGNA et al., 2013).

As formigas Brachyponera chinensis, Brachyponera sennaarensis, e N. goeldii são implicadas comumente em acidentes decorrentes de ferroadas, onde a peçonha é responsável de quadros de anafilatoxia nas vítimas (HOFFMAN, 2010). $\mathrm{Na}$ peçonha de B. sennaarensis, foram identificadas proteínas com massa molecular de 16 e $24 \mathrm{kDa}$, caracterizadas como ligantes IgE. A proteína de $24 \mathrm{kDa}$ foi caracterizada como similar ao antígeno de mesmo peso molecular descrito para S. invicta (REUNALA et al., 2005) e também isolado da peçonha de B. chinensis (LEE et al., 2009). Tal antígeno já foi sequenciado e sua estrutura tridimensional determinada por técnicas cristalográficas (HOFFMAN, 2010). Manso et al. (2010), também através da técnica de eletroforese bidimensional, identificaram na peçonha de $N$. goeldii quatro proteínas ligantes de IgE com peso entre 30 e $45 \mathrm{kDa}$.

\section{Atividade biológica}

Peçonhas de formigas que possuem grande quantidade de alcaloides na sua composição foram até o momento bem estudadas, particularmente as produzidas por espécies dos gêneros Monomorium sp. e Solenopsis sp. (JONES et al., 1999; DESLIPPE; GUO, 2000; CHEN; FADAMIRO, 2009). Em geral, as peçonhas de formigas dos grupos Poneroide e Formicoide são ricas em compostos proteicos. Os Poneroides apresentam em sua composição grande quantidade de peptídeos, especialmente nas peçonhas da subfamília Ponerinae. Peptideos também estão sendo isolados e caracterizados a partir da peçonha de formigas do grupo Formicoide, nas subfamílias Ectatomminae, Myrmeciinae, Myrmicinae e Pseudomyrmecinae (PLUZHNIKOV et al., 1999, WU et al., 1998; SZOLAJSKA et al., 2004; BOUZID et al. 2013; COLOGNA et al., 2013; TOUCHARD et al. 2014a, b, c; AILI et al., 2014).

\section{i) Organismos não Formicidae}

Os estudos mais extensivos de caracterização de peçonhas, dentro dos himenópteros, são voltados para as abelhas sociais, especialmente Apis, e diversas vespas sociais (Vespula, Vespa e Polistes) (SANTANA, 2008). Outros organismos também tiveram sua peçonha bastante estudada, e alguns com comprovada bioatividade. Neste grupo, incluem-se gastrópodes, aranhas, serpentes, lagartos, escorpiões e anêmonas marinhas. O captopril, por exemplo, uma droga anti-hipertensiva empregada em medicina, foi isolada da peçonha da serpente Bothrops jararaca (LEWIS; GARCIA, 2003).

Tais atividades biológicas nem sempre se mostram benéficas e isso depende do mecanismo de ação e do organismo no qual a peçonha é inoculada. Por exemplo, a peçonha do escorpião (Tityus serrulatus) possui atividade imunomodulatória sobre macrófagos murinos, interferindo na produção e liberação de determinadas citocinas e óxido nítrico (PETRICEVICH et al., 2007). O mesmo efeito também foi demonstrado em macrófagos expostos aos venenos de outros animais: vespas (PONVERT et al., 2001), abelhas (JANG et al., 2005) e aranhas (ZANCHET et al., 2004).

Proteínas alergênicas também foram isoladas na peçonha da abelha Apis mellifera carnica como também três novas proteínas (PEIREN et al., 2005). Estas proteínas alergênicas (ou alérgenos) apresentam a propriedade de sensibilizar, ou seja, induzir o sistema imune a produzir anticorpos com elevada especificidade, desencadeando sintomas alérgicos em pacientes sensibilizados (HOFFMAN, 2006; AALBERSE, 2000). Os peptídeos bioativos e as proteínas são os principais responsáveis por edema, dores prolongadas, eritema, reações alérgicas e sistêmicas (LORENZI, 2002).

A melitina é um peptídeo potencialmente tóxico e com grande atividade hemolítica, que foi isolado da peçonha de A. mellifera (SCIANI et al., 2010). Acredita-se que a importância da melitina nas peçonhas pode estar relacionada a sua capacidade de ativar ou inativar peptídeos (LEE et al., 2007; PEIREN et al., 2008).

Além disso, algumas peçonhas apresentam atividade hemolítica e/ou citotóxica, induzindo morte, geralmente por apoptose, em células humanas (BERNHEIMER et al., 1980; NUNES et al., 2012).

Uma proteína foi identificada no veneno de vespa Cyphononyx dorsalis apresentando efeito paralítico sob suas presas (YAMAMOTO et al., 2007). Na peçonha das vespas solitárias Eumenes pomiformis e Orancistrocerus drewseni foram 
identificadas mais de 20 proteínas, sendo a arginina quinase a proteína predominante (BAEK; LEE, 2010).

\section{ii) Formicidae não Poneromorfas}

Diversas peçonhas e toxinas de formigas têm propriedades antimicrobianas, como a solenopsina A, um alcaloide isolado da peçonha de S. invicta que inibe sinalização intracelular na bactéria Gram-negativa Pseudomonas aeruginosa (PARK et al., 2008). O alcaloide piperideína isolado da peçonha de S. invicta exibe atividade antifúngica (DAI et al., 2011). A isosolenopsina A, outro alcaloide isolado desta mesma formiga, é um potente inibidor seletivo da isoforma neuronal da óxido nítrico-sintase, que pode ter efeito significativo nas reações adversas das ferroadas (YI et al., 2003).

$\mathrm{Na}$ peçonha da formiga australiana Myrmecia pilulosa, foram isolados três peptídeos citotóxicos (pilosulinas 1, 2 e 3) (WU et al., 1998). A pilosulina-1, caracterizada como um potente peptídeo antimicrobiano de amplo espectro, apresenta atividade contra microrganismos padrões e multirresistentes Gram-positivos, Gram -negativos e Candida albicans (PLUZHNIKOV et al., 2006).

O veneno de determinadas espécies de formigas apresenta propriedades citotóxicas, como em Formica rufa (BLUM, 1992), e/ ou ação hemolítica, como em Myrmecia pilosula (MATUSZEK et al., 1992), Pseudomyrmex triplarinus (HINK; PAPPAS; JAWORSKI, 1994), Solenopsis spp. e Myrmica ruginodis (BLUM, 1992), Pogonomyrmex barbatus (BERNHEIMER et al., 1980), Myrmecia pilosula (MATUSZEK et al., 1992). As concentrações mínimas de hemólise são variáveis e, dentro de um mesmo gênero, pode haver uma grande discrepância. Por exemplo, são necessárias 500 unid./mg da peçonha seca de Pogonomyrmex barbatus para que haja 50\% de hemólise (BERNHEIMER et al., 1980) [comparativamente, são necessárias apenas 10 unid./ $\mathrm{mg}$ da peçonha seca de Paraponera clavata para obter o mesmo valor de hemólise (SCHMIDT et al., 1984)]. Segundo Matuszek et al. (1992), é necessária uma concentração de $65 \mathrm{ug} / \mathrm{mL}$ da peçonha da formiga Myrmecia pilosula para gerar $12,5 \%$ de hemólise. A hemólise pode ser resultado de um ou mais mecanismos de ação, geralmente ligados à ação de enzima(s). Pode haver uma hemólise direta, onde um ou mais componentes da peçonha agem sobre a membrana eritrocítica, gerando poros e levando à lise. Por exemplo, as fosfolipases clivam os fosfolipídeos da membrana, formando canais e proteínas/peptídeos que possuem atividade surfactante, ambos, favorecendo o extravasamento do conteúdo intracelular (HINK et al., 1994; MATUSZEK et al., 1992). A hemólise indireta pode ser, por exemplo, mediada por ação do complemento, onde componentes da peçonha, como metaloproteinases, facilitam a ação das proteínas iniciadoras da cascata de ativação do complemento (TAMBOURGI et al., 2000).

$O$ veneno de várias espécies apresenta outras propriedades interessantes. Na medicina chinesa, por exemplo, extratos de Polyrhachis lamellidens são tidos como anti-inflamatórios e analgésicos (KOU et al., 2005). Da peçonha de Pseudomyrmex triplarinus foram isoladas seis isoformas de uma proteína com ação anti-inflamatória em edema experimental (PAN; HINK, 2000). A pilosulina $5 \mathrm{da}$ peçonha de M. pilosula é capaz de provocar a liberação de histamina (INAGAKI et al., 2008).

Além disso, a peçonha de algumas formigas pode ainda interferir em outros processos imunológicos, criando um quadro pró-inflamatório, agindo sobre a liberação de determinadas citocinas (SANTANA, 2008), ativação do complemento (SHULTZ et al., 1979) e liberação de mediadores lipídicos, como a histamina (LIND, 1982). A maioria dos componentes alergênicos contidos em venenos de formigas inclui compostos de cerca de 30 $\mathrm{kDa}$ (WIESE et al., 2006).

\section{iii) Poneromorfas}

Na subfamília Ectatomminae, a ectatomina, uma toxina isolada de Ectatomma tuberculatum, age na membrana celular formando canais, e age também em canais de cálcio de miocárdio isolado de rato (PLUZHNIKOV et al., 1999).

Na subfamília Paraponerinae, alguns dos componentes responsáveis para a atividade tóxica de venenos têm sido isolados, tais como a poneratoxina, isolada da peçonha da tocandeira (também conhecida como tocandira ou, ainda, tucandeira) Paraponera clavata (SZOLAJSKA, 2004) e cininas neurotóxicas (PIEK, 1991). Além disso, foram encontradas cininas neurotóxicas em peçonhas de diversas formigas, em particular $P$. clavata (PIEK, 1991). Seu veneno possui um neuropeptídeo que afeta os canais de sódio e bloqueia a transmissão 
sináptica no sistema nervoso central do inseto, de maneira dose-dependente (PLUZHNIKOV et al., 2006).

$\mathrm{Na}$ subfamília Ponerinae, efeitos tóxicos de peçonhas e veneno de diversas espécies de formigas em insetos são relatados, principalmente das peçonhas de Ponerinae nos gêneros Brachyponera (B. chinensis, B. sennaarensis), Bothroponera (Bothroponera insularis), Neoponera (Neoponera apicalis, Neoponera obscuricornis [provavelmente Neoponera verenae], Neoponera villosa), Paltothyreus (Paltothyreus tarsatus), Pseudoneoponera (Pseudoneoponera tridentata) (ORIVEL; DEJEAN, 2001; ORIVEL et al., 2001, CRUZ LÓPEZ et al., 1997, NIKBAKHTZADEH et al. 2009, MASCHWITZ et al. 1981). A indução de apoptose celular foi identificada por BADR et al. (2012) na peçonha de B. sennaarensis frente a células de linhagem tumorais.

Em Neoponera goeldii foram isolados ponericinas, peptídeos que apresentam atividades hemolítica, inseticida contra larvas de gafanhotos, e antimicrobiana, contra bactérias Gram-positivas e Gram-negativas (ORIVEL; DEJEAN, 2001; PLUZHNIKOV et al., 2006). O ensaio antibacteriano utilizando a peçonha bruta da formiga $N$. goeldii demonstrou forte ação contra bactérias gram-positivas (Staphylococcus aureus CIP 677, S. aureus LMA, entre outros) e gram-negativas (Escherichia coli CIP 548, E. coli L, Klebsiella pneumoniae CIP 8291, Pseudomonas aeruginosa CIP A22), a uma concentração de $30 \mu \mathrm{g}$ (peçonha bruta liofilizada)/ $\mu \mathrm{L}$ (ORIVEL et al., 2001).

A peçonha de B. sennaarensis afeta a viabilidade celular de células de linhagem tumoral de câncer de mama, de maneira dose-dependente, ao passo que não afeta a viabilidade de células normais do epitélio de mama, nem de células mononucleares de sangue periférico (BADR et al., 2012). Além disso, apresenta atividade anti-inflamatória in vivo (DKHIL et al. 2010). Kim et al. (2001) demonstraram a presença um ligante específico de IgE na anafilaxia causada por B. chinensis.

A poneratoxina de $P$. clavata também tem atividade neurotóxica (SZOLAJSKA et al., 2004), sendo capaz de afetar a transmissão neuromuscular em ratos, age pré- e pós-sinapticamente (HENDRICH et al., 2002). Da peçonha de Dinoponera quadriceps, foram isoladas neurotoxinas com potencial pró e anticonvulsivante em modelo murino (NÔGA et al., 2012, COLOGNA et al., 2013).

\section{Referências}

AALBERSE, R. C. Structural Biology of Allergens. Journal of Allergy and Clinical Immunology, v.106, n.2, p. 228-238, 2000.

AILI, S. R.; TOUCHARD, A.; ESCOUBAS, P.; PADULA, M.; ORIVEL, J.; DEJEAN, A.; NICHOLSON. G.M. Diversity of peptide toxins in stinging ant venoms. Toxicon, v. 92, p. 166-178, 2014.

BADR, G.; GARRAUD, O.; DAGHESTANI, M.; AL-KHALIFA, M. S.; RICHARD, Y. Human breast carcinoma cells are induced to apoptosis by samsum ant venom through an IGF-1-dependant pathway, PI3K/AKT and ERK signaling. Cellular immunology, v. 273, n. 1, p. 10-6, 2012.

BAEK, J. H.; LEE, S. H. Identification and characterization of venom proteins of two solitary wasps, Eumenes pomiformis and Orancistrocerus drewseni. Toxicon, v. 56, n. 4, p. 554-562, 2010.

BERNHEIMER, A. W.; AVIGAD, L. S.; SCHMIDT, J. O. A hemolytic polypeptide from the venom of the red harvester ant, Pogonomyrmex barbatus. Toxicon, v. 18, n. 3, 271-278, 1980 .

BLUM, M. S. Ant venoms: chemical and pharmacological properties. Journal of Toxicology Toxin Reviews, v. 11, p. 115-64, 1992.

BOUZID, W., KLOPP, C., VERDENAUD, M., DUCANCEL, F., VETILLARD, A. Profiling the venom gland transcriptome of Tetramorium bicarinatum (Hymenoptera: Formicidae): the first transcriptome analysis of an ant species. Toxicon, v. 70, p. 70-81, 2013.

CHEN, L.; FADAMIRO, H. Y. I-Re-investigation of venom chemistry of Solenopsis fire ants. IIIdentification of novel alkaloids in S. invicta. Toxicon, v. 53, p. 479-486, 2009.

COLOGNA, C. T., CARDOSO, J. S., JOURDAN, E., DEGUELDRE, M., UPERT, G., GILLES, N., UETANABARO, A. P. T. ; COSTA NETO, E. M., THONART, P. ; PAUW, E., QUINTON, L. Peptidomic comparison and characterization of the major components of the venom of the giant ant Dinoponera quadriceps collected in four different areas of Brazil. Journal of Proteomics, v. 94, p. 413422, 2013.

CRUZ LÓPEZ, L. C.; MORGAN, E. D. Explanation of the bitter taste of the venom of the ponerine ant Pachycondyla apicalis. Journal of Chemical Ecology, v. 23, p. 705-12, 1997. 
DAI, L.; JACOB, M. R.; KHAN, S. I.; KHAN, I. A.; CLARK, A.M. Synthesis and antifungal activity of natural product-based 6-Alkyl-2,3,4,5tetrahydropyridines. Journal of Natural Products, v. 74, n. 9, p. 2023-2026, 2011.

\section{DESBOIS A. P., LANG S., GEMMELL C. G., COOTE}

P. J. Surface disinfection properties of the combination of an antimicrobial peptide, ranalexin, with an endopeptidase, lysostaphin, against methicillinresistant Staphylococcus aureus (MRSA). Journal of Applied Microbiology, v.108, p.723-730, 2010.

DESLIPPE, R. J.; GUO, Y. J. Venom alkaloids of fire ants in relation to worker size and age. Toxicon. v.38, p.223$232,2000$.

DIZ, A. P., MARTINEX, M., ALVAREZ, E. R. Proteomics in evolutionary ecology: linking the genotype with the phenotype. Molecular Ecology, v.21, p.1060-1080, 2012.

DKHIL M, ABDEL-BAKI A, AL-QURAISHI S, ALKHALIFA M. Anti-inflammatory activity of the venom from samsum ant Pachycondyla sennaarensis. African Journal of Pharmacy and Pharmacology; 4:115-118, 2010 .

DOMON, B.; AEBERSOLD, R. Challenges and opportunities in proteomics data analysis. Molecular; Cellular Proteomics, v. 5, p. 1921-1926, 2006.

ESCOUBAS, P.; KING. G. F. Venomics as a drug discovery platform. Expert Review Proteomics, v. 6, n. 3, 221-224, 2009.

FENNELL, J.; SHIPMAN, W. e COLE, L. Antibacterial action of a bee venom fraction (melittin) against a penicillin-resistant staphylococcus and other microorganisms. Research Development Technology Reports, v. 5, p. 1-13, 1967.

FOX, J. W.; SERRANO, S. M. Exploring snake venom proteomes: multifaceted analyses for complex toxin mixtures. Proteomics, v. 8, p. 909-920, 2008.

GEORGIEVA, D. , SEIFERT, J., OHLER, M., BERGEN, M. V., SPENCER, P., ARNI, R. K., GENOV, N., CHRISTIAN, B.Pseudechis australis Venomics: Adaptation for a Defense against Microbial Pathogens and Recruitment of Body Transferrin. Journal of Proteome Research, v. 10, p. 2440-2464, 2011.

GRAAF, D. C., AERTS, M., DANNEELS, E., DECREESE, B. Bee, wasp and ant venomics pave the way for a component-resolved diagnosis of sting allergy. Journal of Proteomics, v. 72, p. 145-154, 2009.
HADDAD JUNIOR, V.; CARDOSO, J. L. C.; MORAES, R. H.P. Description of an injury in a human caused by a false tocandira (Dinoponera gigantea, Perty, 1833) with a revision on folkloric, pharmacological and clinical aspects of the giant ants of the genera Paraponera and Dinoponera (sub-family Ponerinae). Revista do Instituto de Medicina Tropical de São Paulo, v. 47, n.4, p 235-238, 2005.

HENDRICH, A. B., MOZRZYMAS, J.W., KONOPINSKA, D., SCUKA, M. The effect of poneratoxin on neuromuscular transmission in the rat diaphragm. Cellular and Molecular Biology Letters, v. 7, 195-202, 2002.

HIDER, R. C. Honeybee venom: a rich source of pharmacologically active peptides. Endeavour, new series, v. 12, n. 2, p. 60-62, 1988.

HINK, W. F.; PAPPAS, P. W.; JAWORSKI, D. C. Partial biochemical characterization of venom from the ant Pseudomyrmex triplarinus. Toxicon, v. 32, n. 7, p. 763772, 1994.

HOFFMAN, D. R. Allergens in Hymenoptera venom XXII: comparison of venoms from two species of imported fire ants, Solenopsis invicta and richteri. Journal of Allergy and Clinical Immunology, v. 85, p. 988-996, 1990.

HOFFMAN, D. R. Hymenoptera venom allergens. Clinical Reviews in Allergy; Immunology, v. 30, n. 2, p. 109-128, 2006.

HOFFMAN, D. R. Allergens in Hymenoptera venom XXIV: the amino acid sequences of imported fire ant venom allergens Sol i II, Sol i III and Sol i IV. Journal of Allergy and Clinical Immunology, v. 91, p. 71-78, 1993.

HOFFMAN, D. R. Ant venoms. Current Opinion in Allergy and Clinical Immunol., v. 10, 342-346, 2010.

HOFFMAN, D. R.; DOVE, D. E.; JACOBSON, R. S. Allergens in Hymenoptera venom XX. Isolation of four allergens from imported fire ant (Solenopsis invicta) venom. Journal of Allergy and Clinical Immunology, v. 82, p. $818-827,1988$.

HOFFMAN, D. R.; SAKELL, R. H.; SCHMIDT, M. Sol i 1 , the phospholipase allergen of imported fire ant venom. Journal of Allergy and Clinical Immunology, v. 115, p. 611-616, 2005.

HÖLLDOBLER, B.; WILSON, E. O. The Ants. Harvard University Press, Cambridge, Massachusetts, 732 p., 1990.

INAGAKI, H., AKAGI, M., IMAI, H. T., TAYLOR, R. W., WIESE, M. D., DAVIES, N. W., KUBO T Pilosulin 5, a novel histamine-releasing peptide of the Australian ant, Myrmecia pilosula (jack jumper ant). Archives of Biochemistry and Biophysics, v. 477, p. 411-416, 2008. 
JANG, H. S. KIM, S. K., HAN, J. B., AHN, H. J., BAE, H., MIN, B. I. Effects of bee venom on the proinflammatory responses in RAW 264.7 macrophage cell line. Journal of Ethnopharmacology, v. 99, p. 157160, 2005.

JOHNSON, S. R. COPELLO, J. A., EVANS, M. S., SUAREZ, A. V. A biochemical characterization of the major peptides from the venom of the giant Neotropical hunting ant Dinoponera australis. Toxicon. v.55, p.702-710, 2010.

JONES, T. H., GORMAN, J. S. T., SNELLING, R. R., DELABIE, J. H. C., BLUM, M. S., GARRAFO, H. M., JAIN, P., DALY, J. W., SPANDE, T. F. Further alkaloids common to ants and frogs: decahydroquinolines and a quinolizidine. Journal of Chemical Ecology, 1999, v. 25, n. 5, p.1179-1193.

KIM, S. S., PARK, H. S., KIM, H. Y., LEE, S. K., NAHM, D. H. Anaphylaxis caused by the new ant, Pachycondyla chinensis: demonstration of specific IgE and IgE-binding components. Journal of Allergy and Clinical Immunology, v. 107, n. 6, p. 1095-1099, 2001

KOU, J., NI, Y., LI, N., WANG, J., LIU, L., JIANG, Z. $\mathrm{H}$. Analgesic and anti-inflammatory activities of total extract and individual fractions of Chinese medicinal ants Polyrhachis lamellidens. Biological; Pharmaceutical Bulletin, v. 28, p. 176-180, 2005.

KUHN-NENTWIG, L.; STOCKLIN, R.; NENTWIG, W. Venom Composition and Strategies in Spiders: Is Everything Possible? Advances in Insect Physiology, v. 60, p. 1-86, 2011.

LEE, E. K., JEONG, K. Y., LYU, D. P., LEE, Y. W., SOHN, J. H., LIM, K. J. Characterization of the major allergens of Pachycondyla chinensis in ant sting anaphylaxis patients. Clinical; Experimental Allergy, v. 39, p. 602-607, 2009.

LEE, V. S. Y., TU, W. C., JINN, T. R., PENG, C. C., LIN, L. J., TZEN, J. T. C. Molecular cloning of the precursor polypeptide of mastoparan $B$ and its putative processing enzyme, dipeptidyl peptidase IV, from the black-bellied hornet Vespa basalis. Insect Molecular Biology, v.16, p. 231-237, 2007.

LEWIS, R. J.; GARCIA, M. L. Therapeutic potential of venom peptides. Nature Reviews, v. 2, 790-802, 2003.

LIND, N. K. Mechanism of action of fire ant (Solenopsis) venoms. I. Lytic release of histamine from mast cells. Toxicon, v. 20, n. 5, p. 831-840, 1982.
LORENZI, C. C. B. Estudo Estrutural de mastoparanos isolados de vespas solitárias. Dissertação (Mestrado em Biofísica Molecular - Instituto de Biociências, Letras e Ciências Exatas), Universidade Estadual Paulista, São José do Rio Preto, 2002.

MANSO, E. C., CROCE, M, PINTO, J. R. A. S., SANTOS, K. S., SANTOS, L. D,. Anaphylaxis due to Pachycondyla goeldii ant: a case report. Journal of Investigational Allergology and Clinical Immunology, v. 20, n. 4, p. 352-363, 2010.

MASCHWITZ, U., JESSEN, K., MASCHWITZ, E. Foaming in Pachycondyla: a defence mechanism in ants. Behavioral Ecology and Sociobiology, V.40, p. 87-93, 1981.

MATUSZEK, M. A., WAYNE, C. H., STRUAN, K. S., ROGER, G. K. Pharmacological studies of jumper ant (Myrmecia pilosula) venom: evidence for the presence of histamine, and haemolytic and eicosanoid-releasing factors. Toxicon, v. 30, n. 9, p. 1081-1091, 1992.

NIKBAKHTZADEH, M. R., AKBARZADEH, K., TIRGARI, S. Bioecology and chemical diversity of abdominal glands in the iranian samsum ant Pachycondyla sennaarensis (Formicidae: Ponerinae). Journal of Venomous Animals and Toxins including Tropical Diseases, V. 15, p. 526-36, 2009.

NÔGA, D. A. M. F., CAGNI, F. C.; SANTOS, J. R.; AZEVEDO, D. L. O.; ARAÚJO, A. Pro- and anticonvulsant effects of the ant Dinoponera quadriceps venom in mice. I Workshop Peçonha de Formigas: Pesquisas e Perspectivas, Ilhéus - Brasil, 2012.

NUNES, E. S.; SOUZA M. A.; VAZ, A. F.; SILVA, T. G.; AGUIAR, J. S.; BATISTA, A. M.; GUERRA, M. M.; GUARNIERI, M. C.; COELHO, L. C.; CORREIA, M. T. Cytotoxic effect and apoptosis induction by Bothrops leucurus venom lectin on tumor cell lines. Toxicon, v. 59, n. v. 7-8, p. 667-671, 2012.

ORIVEL, J., REDEKER, V., LE CAER, J. P., KRIER, F., REVOL-JUNELLES, A. M., LONGEON, A. Ponericins, new antibacterial and insecticidal peptides from the venom of the ant Pachycondyla goeldii. The Journal of Biological Chemistry, v. 276, n. 21, p. 17823-17829, 2001.

ORIVEL, J.; DEJEAN, A. Comparative effect of the venoms of the ants of the genus Pachycondyla (Hymenoptera: Ponerinae). Toxicon, v. 39, p. 195-201, 2001.

PALMA, M. S., Insect venom peptides. Handbook of Biologically Active Peptides. $1^{\text {a }}$ ed. Oxford, Academic Press, cap. 56, p.409-416, 2006. 
PAN, J.; HINK, W. F. Isolation and characterization of myrmexins, six isoforms of venom proteins with anti-inflammatory activity from the tropical ant, Pseudomyrmex triplarinus. Toxicon, v. 38, p. 1403-1413, 2000.

PARK, J., GUNNAR, F. KAUFMANN, J., PHILLIP B, JACK, L., A. Solenopsin A, a venom alkaloid from the fire ant Solenopsis invicta, inhibits quorum-sensing signaling in Pseudomonas aeruginosa. The Journal of Infectious Diseases, v. 198, 1198-1201, 2008.

PEIREN, N., DE GRAAF, D. C., VANROBAEYS, F., DANNEELS, E. L., DEVREESE, B., VAN BEEUMEN, J., JACOBS, F. J. Proteomic analysis of the honey bee worker venom gland focusing on the mechanisms of protection against tissue damage. Toxicon, v. 52, n. 1, p. 72-83, 2008.

PETRICEVICH, V. L., HERNÁNDEZ CRUZ, A., CORONAS, F. I., POSSANI, L. D. Toxin gamma from Tityus serrulatus scorpion venom plays an essential role in immunomodulation of macrophages. Toxicon, v. 50, p. 666-675, 2007.

PIEK, T. Neurotoxic kinins from wasp and ant venoms. Toxicon, v. 29, n. 2, p. 139-149, 1991.

PIEK, T., DUVAL, A., HUE, B., KARST, H., LAPIED. B., MANTEL, P., NAKAJIMA. T., PELHATE, M., SCHMIDT, J. O. L. Poneratoxin, a novel pepetide neurotoxin from the venom of the ant, Paraponera clavata. Comparative Biochemistry and Physiology, v. 99, p. 487-495, 1991.

PINTO, J. R. A. S., FOX, E. G. P., SAIDEMBERG, D.M., SANTOS. L. D. Proteomic view of the venom from the fire ant Solenopsis invicta Buren. Journal of Proteome Research, v. 11, n. 9, p. 4643-53, 2012.

PLUZHNIKOV, K., BOCHAROV, D. N., KONONOVA, N. V., SUKHANOV, S. V., BALASHOVA, T. A., ARSENIEV,A.S., GRISHIN, E. V. Identification and structural analysis of a glycophospholipid component from the venom of ant Paraponera clavata. Russian Journal of Bioorganic Chemistry, v. 32, n. 5, p. 478-484, 2006.

PLUZHNIKOV, K., NOSYREVA, E., SHEVCHENKO, L., KOKOZ, Y., SCHMALZ, D., HUCHO, F., GRISHIN, E. Analysis of ectatomin action on cell membranes.

European Journal of Biochemistry/FEBS, v. 262, n. 2, p. 501-506, 1999.

PONVERT, C., LE COURVOISIER, C., WEILL, B., BLOCH, E., PAUPE, J., SCHEINMANN, P. Kinetcs of plasma cytokine levels in children hyposensitized with wasp venom. Cytokine, v. 15, n. 4, p. 229-231, 2001.
PUPO, M. T.; GALLO, M. B. C. Biologia química: uma estratégia moderna para a pesquisa em produtos naturais. Química Nova. v. 30, n. 6, p. 1446-1455, 2007.

RATES, B. et al. Peptidomic dissection of the skin secretion of Phasmahyla jandaia (Bokermann and Sazima, 1978) (Anura, Hylidae, Phyllomedusinae). Toxicon, v. 57, n. 1, p. 35-52, 2011.

RATES, B., BEMQUERER, M. P., RICHARDSON, M., BORGES, M. H., MORALES, R. A., DE LIMA, M. E., PIMENTA, A. M. Venomic analyses of Scolopendra viridicornis nigra and Scolopendra angulata (Centipede, Scolopendromorpha): shedding light on venoms from a neglected group. Toxicon, v. 49, n. 6, p. 810-26, 2007.

REUNALA, T. et al. Characterization of IgE-binding allergens in Samsum ant venom. Journal of Allergy and Clinical Immunology, v. 115, p. 115-108., 2005.

RIFFLET, A., SABINE. G., NATHAN. T., JÉRÔME, O., JÉRÔME, L. C. Identification and characterization of a novel antimicrobial peptide from the venom of the ant Tetramorium bicarinatum. Peptides, v. 38, p. 363-378, 2012.

ROCHA, T. L., BRUMMER-KOVENKONTIO, H., SAARIEN, K., RASANEN, L., LESTRINGANT, Eletroforese bidimensional e análise de proteomas. Embrapa, Comunicado Técnico n. 137, p. 1-12, 2007.

SANTANA, F. A. Imunogenicidade do veneno de Dinoponera australis (Hymenoptera, Formicidae, Ponerinae). Tese (Doutorado em Genética e Bioquímica) - Instituto de Genética e Bioquímica, Universidade Federal de Uberlândia, Uberlândia, Minas Gerais, 2008.

SANTOS, L. D., PIERONI, M., MENEGASSO, A. R. S., PINTO, J. R. A. S., PALMA, M. S. Brown Recluse Spider Venom: Proteomic Analysis and Proposal of a Puta- tive Mechanism of Action. Protein and Peptide Letters, v. 16, n. 8, p. 933-943, 2009.

SANTOS, L. D., PIERONI, M., MENEGASSO, A. R. S., PINTO, J. R. A. S., PALMA, M. S. A new scenario of bioprospecting of Hymenoptera venoms through proteomic approach. Journal of Venomous Animals and Toxins including Tropical Diseases, v. 17, n. 4, p. 364-377, 2011.

SANTOS, L. D.; PALMA, M. S. Análise proteômica de venenos de himenópteros e a tecnologia de alérgenos recombinantes. p. 1-3, In: CASTRO, F. F. M.; PALMA, M. S. (Coord.). Alergia a Venenos de Insetos, 1 ed. (Série Alergias). Barueri: Manole. 231 p., 2009.

SCHMIDT, J. O.; BLUM, M. S. A harvester ant venom: chemistry and pharmacology. Science, v. 200, 10641066, 1978b. 
SCHMIDT, J. O.; BLUM, M. S. The biochemical constituents of the venom of the harvester ant, Pogonomyrmex badius. Comparative Biochemistry and Physiology - Part C: Toxicology; Pharmacology, v. 61, p. $239-247,1978$ a.

SCHMIDT, J. O.; BLUM, M. S.; OVERAL, W. L. Hemolytic Activities of Stinging Insect Venoms. Archives of Insect Biochemistry and Physiology, p. 155-160, 1984.

SCHWARTZ, E. F., CAMARGOS, T. S., ZAMUDIO, F. Z., SILVA, L. P., BLOCH JR, C., CAIXETA, F. Mass spectrometry analysis, amino acid sequence and biological activity of venom components from the Brazilian scorpion Opisthacanthus cayaporum. Toxicon, v. 51, p. 1499-1508, 2008.

SCIANI, J. M., MARQUES-PORTO, R., LOURENÇO JR, A., ORSI, R. O., FERREIRA JUNIOR, R. S., BARRAVIERA, B., PIMENTA, D. C. Identification of a novel melittin isoform from Africanized Apis mellifera venom. Peptides, v. 31, n.8, p.1473-1479, 2010.

SHULTZ, D. R., ARNOLD, P. I., WU, M. C. Isolation and partial characterization of a polysaccharide in ant venom (Pseudomyrmex sp.) that activates the classical complement pathway. Molecular Immunology, v. 16, p. 253-264, 1979.

SILVA e SILVA, A. M.; CORRÊA, G. C.; REIS, E. M. Proteômica - uma abordagem funcional do estudo de genoma. Saúde e Ambiente em Revista, v. 2, n.2 2, p. 1-10, 2007.

STEEN, C. J., JANNIGER, C. K., SCHUTZER, S. E. Insect sting reactions to bees, wasps, and ants. International Journal of Dermatology, v. 44, p. 91-94, 2005.

SZOLAJSKA, E. POZNANSKI, J., FERBER, M. L., MICHALIK, J., GOUT, E., FENDER, P., BAILLY, I., DUBLET, D. Poneratoxin, a neurotoxin from ant venom. Structure and expression in insect cells and construction of a bio-insecticide. European Journal of Biochemistry, v. 271, p. 2127-2136, 2004.

TAMBOURGI, D. V., MORGAN, B. P., ANDRADE, R. M. G., MAGNOLI, F. C., BERG, C. W. Loxosceles intermedia spider envenomation induces activation of an endogenous metalloproteinase, resulting in cleavage of glycophorins from the erythrocyte surface and facilitating complement-mediated lysis. Blood, v. 95, p. 683-691, 2000.

TORRES, A. F. C., HUANG, C., CHONG, C.M., LEUNG, S. W., PRIETO-DA-SILVA, Á. R. B.'HAVT, A., QUINET, P., MARTINS, A. M. C.,' , SIMON, M. Y. L., I RÁDIS-BAPTISTA, G. Transcriptome Analysis in Venom Gland of the Predatory Giant Ant Dinoponera quadriceps: Insights into the Polypeptide Toxin Arsenal of Hymenopterans. Plos One, v. 9, n. 1, p. 1-17, 2014.
TOUCHARD, A.; DAUVOIS, M.; KOH, J. M. S.; PETITCLERC, F; DEJEAN, A.; NICHOLSON, G. M.; ORIVEL, J.; ESCOUBAS, P. Elucidation of the un explored biodiversity of ant venom peptidomes via MALDI-POF mass spectrometry and its application for chemotaxonomy. Proteomics, v. 13, p. 105-217, 2014 b.

TOUCHARD, A.; KOH, J. M. S.; AILI, S. R.; DEJEAN, A.; NICHOLSON, G. M.; ORIVEL, J.; ESCOUBAS, P. The complexity and structural diversity of venom peptidomes is revealed by mass spectrometry profiling. Rapid Communications in Mass Spectrometry, v. 29, p. 385-396, 2014a.

TOUCHARD, A.; LABRIÈRE, N.; ROUX, O.; PETITCLERC, F; ORIVEL, J.; ESCOUBAS, P.; KOH, J.M.S.; NICHOLSON, G.M.; DEJEAN, A. Venom toxicity and composition in three Pseudomyrmex ant species having different nesting modes. Toxicon, v. 88, p. 67-76, 2014c.

VALLES, S. M.; PEREIRA, R. M. Solenopsis invicta transferrin: cDNA cloning, gene architecture, and upregulation in response to Beauveria bassiana infection. Gene. v.358, p. 60-66, 2005.

VON SICARD, N. A. E.; CANDY, D. J.; ANDERSON, M. The biochemical composition of venom from the pavement ant (Tetramorium caespitum L.). Toxicon, v. 27, n. 10, p. 1127-1133, 1989.

WHEELER, W. M., 1910. Ants: their Structure, Development and Behavior, Columbia University Press, New York, xxv + 663 p.

WIESE, M. D., CHATAWAY, T. K., DAVIES, N.W., MILNE, R.W., BROWN, S. G.A., GAI, W. P., HEDDLE, R. J. Proteomic analysis of Myrmecia pilosula (Jack jumper) ant venom. Toxicon, v. 47, n. 2, p. 208-217, 2006.

WILKINS, M. R.; PASQUALI, C.; APPEL, R. D. From proteins to proteomes: large scale proteina identification by two-dimensional electrophoresis and amino acid analysis. Biotechnology, v. 14, p. 61-65, 1996.

WILLIAMS, K. L.; HOCHSTRASSER, D. F. Proteome Research: New Frontiers in Functional Genomics, Springer, 245 p., 1997.

WU, Q. X., KING, M. A., DONOVAN, G. R., BALDO, B. A. Cytotoxicity of pilosulin 1, a peptide from the venom of the jumper ant Myrmecia pilosula. Biochimica et Biophysica Acta, v. 1425, n. 1, p. 74-80, 1998.

YAMAMOTO, T., ARIMOTO, H., KINUMI, T., OBA, Y., UEMURAY. Identification of proteins from venom of the paralytic spider wasp, Cyphononyx dorsalis. Insect Biochemistry and Molecular Biology. v.37, p.278-286, 2007. 
YI, G.B., MC CLENDON, D., DESAIAH, D., GODDARD, J., LISTER, A., MOFFITT, J., VANDER MEER, R. K., SHAZO, R., LEE, K.S., ROCHKHOLD, $\mathrm{R}$. W. Fire ant venom alkaloid, isosolenopsin A, a potent and selective inhibitor of neuronal nitric oxide synthase. International Journal of Toxicology, v. 22, n. 2, p. 81-86, 2003.

YUN, E. Y., LEE, J. K., KWON, O. Y., HWANG, J. S., KIM, I., KANG, S. W., LEE, W. J., DING, J. L., GOO, T.W. Bombyx mori transferrin: genomic structure, expression and antimicrobial activity of recombinant protein. Developmental and comparative

Immunology, v. 33, n. 10, p. 1064-1069, 2009.
ZANCHET, E. M.; LONGO, I.; CURY, Y. Involvement of spinal neurokinins, excitatory amino acids, proinflammatory cytokines, nitric oxide and prostanoids in pain facilitation induced by Phoneutria nigriventer spider venom. Brain Research, v. 1021, p. 101-111, 2004. 